INPLASY

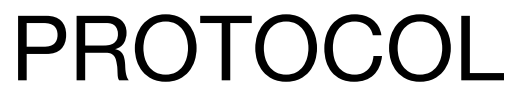

To cite: Platt et al. Protocol: A systematic review of the economic impact of surgery in low income countries. Inplasy protocol 202040167. doi: 10.37766/inplasy2020.4.0167

Received: 24 April 2020

Published: 24 April 2020

Corresponding author: Caris Grimes

caris.grimes@kcl.ac.uk

Author Affiliation:

King's College London

Support: None.

Review Stage at time of this submission: Data extraction.

Conflicts of interest:

None.

\section{Protocol: A systematic review of the economic impact of surgery in low income countries}

\author{
Platt, E1; Doe, M²; Kim, N3; Grimes, CE4.
}

Review question / Objective: What is the economic impact of surgical disease and intervention within low income countries? This will be subdivided into the macro-economic impact at Country/Region/District level and the microeconomic impact at individual/family level and is likely to form the basis of 2 reviews.

Condition being studied: All surgical conditions.

Rationale: With increasing evidence of and interest in the field of global surgery within the wider field of global health, this systematic review will aim to bring together all studies pertaining to costs outside of the facility where surgical care is provided. It will look firstly at the costs to individuals and households, and secondly costs to countries and regions of the world.

INPLASY registration number: This protocol was registered with the International Platform of Registered Systematic Review and Meta-Analysis Protocols (INPLASY) on 24 April 2020 and was last updated on 24 April 2020 (registration number INPLASY202040167).

\section{INTRODUCTION}

Review question / Objective: What is the economic impact of surgical disease and intervention within low income countries? This will be subdivided into the macroeconomic impact at Country/Region/ District level and the micro-economic impact at individual/family level and is likely to form the basis of 2 reviews.

Rationale: With increasing evidence of and interest in the field of global surgery within the wider field of global health, this systematic review will aim to bring together all studies pertaining to costs outside of the facility where surgical care is provided. 
It will look firstly at the costs to individuals and households, and secondly costs to countries and regions of the world. Condition being studied: All surgical
conditions.

\section{METHODS}

Search strategy: 1. Pubmed and Medline. 2. Search terms: 1. Surgery (((")surgical procedures, operative"[MeSH Major Topic]) OR "surgery"[MeSH Subheading]) OR "surgery") OR "surgical procedure") OR surgical procedures 45206712 2. LICs

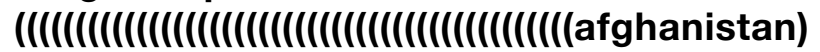
OR benin) OR burkina faso) OR burundi) OR central african republic) OR chad) OR comoros) OR congo) OR eritrea) OR ethiopia) OR gambia) OR guinea) OR guinea-bissau) OR haiti) OR korea, democratic people's republic) OR north korea) OR liberia) OR madagascar) OR malawi) OR mozambique) OR nepal) OR niger) OR rwanda) OR senegal) OR sierra leone) OR somalia) OR south sudan) OR syrian arab republic) OR syria) OR tajikistan) OR tanzania) OR togo) OR uganda) OR yemen) OR zimbabwe))) OR korea[MeSH Major Topic]) OR nepal[MeSH Major Topic]) OR rwanda[MeSH Major Topic]) OR senegal[MeSH Major Topic]) OR somalia[MeSH Major Topic]) OR tanzania[MeSH Major Topic]) OR yemen[MeSH Major Topic]) OR $(\langle(()(()($ (low resource) OR under-resourced) OR resource poor) OR under-developed) OR underdeveloped) OR developing world) OR third world) OR low income) OR developing country) OR developing countries))) OR

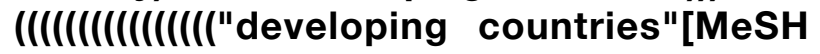
Major Topic]) OR "developing countries/ economics"[MeSH Major Topic]) OR "developing countries") OR developing countries Imic) OR developing country) OR "low resource countries") OR low resource developing country) OR "low income") OR "Imic") OR "Iow middle income") OR low middle income countries) OR low middle income country) OR low middle income countries Imic) OR "third world") OR third world countries) OR third world country)) 600144 3. Step $11 \& 252069$ 4. Econ ()((()(()(("economics"[MeSH Major Topic])
OR "economics/economics"[MeSH Major Topic]) OR "economics/trends"[MeSH Major Topic]) OR "cost benefit analysis"[MeSH Major Topic]) OR "income"[MeSH Major Topic]) OR "income/ economics" [MeSH Major Topic]) OR "economics"[MeSH Subheading]) OR "poverty"[MeSH Major Topic]) OR "poverty") OR "cost") OR "income") OR "expenditure") OR "gdp" 1021451 5. Step 2 $3 \& 410810$ 6. English Filtered 10409.

Participant or population: Studies with data pertaining to low income countries (World Bank Classification).

Intervention: Surgical treatment - any that requires an anaesthetic and includes all surgical disciplines, maternal health etc.

Comparator: N/a.

Study designs to be included: All relevant including qualitative, economic and cohort.

Eligibility criteria: Data from a low income country, related to health economics either individual, household or country, regional; in English.

Information sources: Medline and Pubmed.

Main outcome(s): For individual / household data: Paper; Year of publication; Country; intervention; methodology; direct medical costs; direct non-medical costs; indirect costs; how costs were met; longterm financial impact. For regional / country data: Paper; Year of publication; Country / region; intervention; methodology; results; notes.

Additional outcome(s): n/a.

Data management: Data will be uploaded to Rayyan for review; included papers will then be uploaded onto Rayyan. Data extraction to Microsoft excel spreadsheet.

Quality assessment / Risk of bias analysis: Because the papers will be heterogenous, the following will be used depending on the paper: - SRQR for qualitative studies; STROBE for observational studies; - 
CHEERS for economic studies; A scoring system will be applied to determine whether each study is high, medium or low quality.

Strategy of data synthesis: All data will be placed into excel spreadsheet as described above. A narrative synthesis will be compiled.

Subgroup analysis: Two groups as described above. No subgroups.

Sensibility analysis: N/a.

Language: English.

Country(ies) involved: All low income countries according to the World Bank classification.

Keywords: Low income countries, health economics, surgery, global health, global surgery.

Dissemination plans: Publication in relevant journals.

Contributions of each author:

Author 1 - Esther Platt - TBC.

Author 2 - Matthew Does - TBC.

Author 3 - Na Eun Kim - TBC.

Author 4 - Caris Grimes - TBC. 